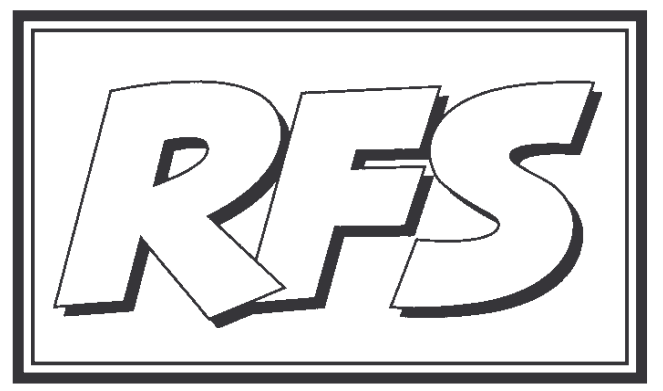

Revista de Fomento Social, 61 (2006), 637-646

\title{
Congreso Internacional de Desarrollo Humano en Madrid. Una crónica
}

— Francis SANTOS CARRILLO y José J. ROMERO RODRÍGUEZ SJ ${ }^{1}$

Del 14 al 16 de noviembre de 2006 tuvo lugar en el Palacio Municipal de Congresos de Madrid (Campo de las Naciones) el I Congreso Internacional sobre Desarrollo Humano². El tema del congreso fue: "Controversia, autocrí

${ }^{1}$ Fundación ETEA para el Desarrollo y la Cooperación (Córdoba).

2 Aunque, creemos que con razón, se ha cuestionado que se trate del "primer" Congreso sobre el tema, ni siquiera en España. Koldo Unceta, de Hegoa, lo cuestionaba en una carta al diario El País del 6 de octubre de 2006: "deseo manifestar que el I Congreso Internacional sobre Desarrollo Humano ya se celebró en Bilbao, en la Universidad del País Vasco, en el año 1999, organizado por el Instituto Hegoa del cual yo era entonces director, asistiendo al mismo varios cientos de profesores e investigadores de universidades españolas y extranjeras. En consecuencia, el evento organizado ahora por el Ayuntamiento de Madrid -sea cual sea su naturaleza- no debería arrogarse tal título". Desgraciadamente es frecuente 
tica y realismo. Retos de hoy. Soluciones de futuro". Según los organizadores el número de congresistas superó las 700 personas.

Los objetivos del Congreso quedaban recogidos en el propio programa del Congreso:

"El Ayuntamiento de Madrid celebra el I Congreso Internacional sobre Desarrollo Humano Madrid 2006 justo quince años después de publicarse el primer Informe de Desarrollo Humano del PNUD y en un momento en el que se aprecia mayor consenso en la sociedad internacional sobre la vigencia y validez del concepto.

Desde 1990 la globalización se ha consolidado creando oportunidades, importantes desafíos y nuevos retos para el Estado y la sociedad civil ante la necesidad de extender sus beneficios a toda la población. Los estudiosos del desarrollo y la economía analizan las causas de la pobreza y la desigualdad para aportar soluciones, pero este esfuerzo intelectual es, a veces, cuestionado por una parte importante de la sociedad, que considera inaceptable el estado actual del desarrollo en el mundo. La globalización dominante no satisface a sectores importantes de población que, a su vez, se globalizan en la protesta para adquirir relevancia y mostrar la amplitud de su desacuerdo.

Con este Congreso la ciudad de Madrid desea proporcionar acogida a una reflexión independiente sobre el estado del desarrollo humano y los nuevos desafíos que se plantean, enfocándolo a través de cinco líneas temáticas fundamentales, sin pretender agotar sus contenidos o limitar debates más extensos, que podrán ser abordados en futuras ediciones del Congreso.

Se abre así un espacio para recoger visiones contrapuestas y experiencias concretas que analicen las diferentes facetas del desarrollo humano a través de personas vincu ladas a la formulación del concepto mismo de desarrollo humano y de abanderados tanto intelectuales como de la acción directa en este campo.

Controversia, autocrítica y realismo han de constituir los referentes intelectuales significativos del Congreso para proyectar hacia el futuro un nuevo orden de buenas prácticas que puedan ser difundidas y aplicadas bajo un enfoque de carácter científico y socialmente ético."

\section{Las sesiones plenarias}

El congreso estuvo estructurado en 4 sesiones plenarias y en sesiones pa ralelas agrupadas en cinco grandes líneas temáticas. Resumimos brevemente primero el contenido de dichas sesiones plenariảspara pasar a continuación a las secciones paralelas o líneas temáticas.

que los organizadores de toda suerte de Congresos, Jornadas y Encuentros se arroguen indebidamente el ser "los primeros".

${ }^{3}$ El resumen de estas sesiones, que se presentó en la sesión plenaria final, estuvo a cargo de Francisco Javier Sota quien había también moderado dichas sesiones. 
En la primera sesión plenaria la conferencia principal estuvo a cargo del profesor Richard Jolly del Instituto de Estudios sobre Desarrollo, Universidad de Sussex, Reino Unido ("Desarrollo humano: retos para el siglo XXI"); intervino como comentarista Alfonso Dubois, del Instituto de Estudios so bre Desarrollo y Cooperación Internacional (HEGOA), Universidad del País Vasco ("El Desarrollo Humano como paradigma alternativo"). En esencia, hubo coincidencia de planteamientos y valoraciones entre el ponente y su comentarista.

Partiendo de la necesidad de un nuevo enfoque de Desarrollo (con objetivos más amplios, no sólo económicos; con perspectivas globales, no sólo nacionales o regionales; con sostenibilidad a largo plazo, no sólo hasta las próximas elecciones), surge el convencimiento cada vez más generalizado de que el crecimiento del PNB no es suficiente como indicador de desarrollo, de ahí que sea necesario el Desarrollo Humano que implica situar a las personas en el centro del desarrollo, ampliar las capacidades humanas y posibilidades de elección, asegurar la democracia y no quedarse solamente en apoyo a los sectores sociales, reducción de la pobreza, inversión en educación y salud, o indicadores como el IDH. Para ello habrá que adoptar unas estrategias de desarrollo humano encaminadas a la ampliación de las capacidades humanas, a asegurar a las poblaciones rurales y urbanas sus oportunidades de participar en decisiones, planificación y acción, a asegurar el acceso a los recursos de las poblaciones más pobres. Es posible: hay ejemplos de éxito. Ambas intervenciones pusieron una vez más de manifiesto el consenso, prácticamente generalizado, que existe con respecto a la valoración sobre el desarrollo mundial: "el sistema es injusto". Cabe, por tanto, pensar que es necesario una mayor capacidad de respuesta por parte de la clase política que atienda este requerimiento de la sociedad civil.

La segunda sesión plenaria estuvo centrada en el tema "Globalización y desarrollo". El ponente principal fue el profesor Rafael Kaplinsky del Instituto de Estudios sobre Desarrollo, Universidad de Sussex, Reino Unido. El título de su ponencia fue: “¿Hasta qué punto puede sostenerse la globalización? ¿Y cuál es la diferencia que aporta China?”. Actuó de comentarista María Blanco, de la Universidad CEU San Pablo y miembro del Instituto Juan de Mariana.

El profesor Kaplinski subrayó los principales retos que plantea la globalización, analizada críticamente: el reto medioambiental y el crecimiento de la desigualdad; es cierto que el capitalismo suele "vencer" en la batalla por la supervivencia porque, esencialmente, logra innovar rápidamente; pero las cadenas de valor globales son intensivas en consumo de energía que, a 
su vez, consume el medio ambiente. En dicho contexto destacó el papel que juegan China e India en todos esos retos, para concluir con una pregunta que es un enorme desafío: “¿es sostenible un futuro como este para la co munidad mundial?".

La respuesta, contundente y radicalmente neoliberal, de la joven profesora Blanco sorprendió al público (como se detectó en el turno de preguntas), tanto por su dogmatismo "del mercado" y su falta de matices como por la dureza de los adjetivos que aplicó a las ideas del ponente. Según ella, en resumen, la globalización no es el problema, es la solución; concluyó significativamente citando a Hayek: "sólo el capitalismo hace posible el bienestar".

En la tercera sesión plenariaPedro Videla, del IESE (Barcelona), desarrolló el tema "Pobreza y desigualdad". Por situarlo en relación a los ponentes de la víspera, podríamos decir que se encontraba más cerca de la profesora Blanco que del profesor Kaplinsky. Se mostró más optimista que los expertos británicos de las dos primeras sesiones plenarias acerca de las posibilidad de vencer la desigualdad y la pobreza. Según sus datos, está disminuyendo la pobreza en términos absolutos, esencialmente porque los grandes países (China) están creciendo deprisa, y está aumentando la desigualdad. Lo que el ponente llamó "la verdad conveniente" es que se están produciendo cambios importantes en productividad y en demografía. Con alta probabilidad las condiciones materiales seguirán mejorando; dentro de un siglo es posible que la mayoría de la humanidad tengo un nivel de vida adecuado; pero la otra parte de la realidad, "la verdad inconveniente" consiste en que no se aprecie crecimiento sustancial en África subsahariana; a no ser que África crezca pronto, la desigualdad va a aumentar a escala mundial. La pregunta es ¿por qué no crece África? ¿Podemos hacer algo? Esta ponencia no tuvo comentarista.

La última conferencia magistral estuvo a cargo de Enrique V. Iglesias, Secretario de las Cumbres Iberoamericanas. Sus reflexiones se centran en la problemática de la pobreza en América Latina; a partir de su vasta ex periencia expone 12 lecciones aprendidas o conclusiones de la praxis que resumimos telegráficamente:

1. El asistencialismo como política social tiene un alcance limitado

2. El crecimiento económico cuenta, pero no es suficiente.

3. La estabilidad es importante; no hay peor impuesto para los pobres que la inflación. 
4. Las crisis financieras también importan.

5. La macroeconomía cuenta, hay que conducir bien las políticas ma croeconómicas.

6. Es esencial la mejora en la educación.

7. Son necesarias políticas de distribución del ingreso; se debe proseguir en la lucha por una mayor igualdad.

8. Es necesario facilitar el acceso de los microempresarios al mercado, como vía para promover el desarrollo empresarial.

9. Hace falta mayor y mejor gasto social.

10. Se necesitan más políticas de familia, al ser el corazón de la estructura social.

11. El proteccionismo comercial recorta los ingresos del agricultor y de los intermediarios.

12. Todo esto implica un buen gobierno y una mayor responsabilidad social de la empresa.

\section{Los seminarios por líneas temáticas}

En las secciones monográficas, el congreso estuvo estructurado en 5 grandes líneas temáticas, cada una con sus correspondientes coordinadores académicos:

1. Migraciones y desarrollo (Jaime Atienza - Fundación Carolina).

2. Desarrollo humano y gobernanza democrática (Carmen Mormeneo Cer tés - Departamento de Cooperación del Ayuntamiento de Madrid).

3. Desarrollo urbano. Ciudad sostenible (José Miguel Fernández Güell - Universidad Politécnica de Madrid).

4. Desarrollo económico y cooperación (José Ma Larrú Ramos - CEU San Pablo - Madrid).

5. Desarrollo rural. Nueva ruralidad (Pedro Caldentey del Pozo - Fundación ETEA - Córdoba).

Los autores de esta crónica participamos únicamente en la línea temáti ca V, la cual -como las cuatro restantes-se desarrolló en 5 seminarios de 
aproximadamente 2 horas cada uno. Presentamos a continuación una síntesis de dichos seminarios.

Ante todo, constatamos que el seminario ha cumplidos dos de los princi pales objetivos del Congreso.Ha habido controversia, autocrítica y realismo $\mathrm{y}$ ha recogido voces diversas y abierto espacio a algunos debates y regiones a los que no siempre tenemos acceso.

El Seminario 1 estuvo centrado en el tema "Agricultura y comercio internacional", con intervenciones de Dipak Patel (exministro de Comercio de Zambia) y Juana Galván (Costa Rica) y moderada por Carlos Tió (Universidad Politécnica de Madrid). Resumiendo mucho, podemos decir que, tanto en las exposiciones como en el diálogo siguiente, estuvieron de acuerdo en que, aunque el comercio no resuelve todas las fracturas y problemas de un país o bloque regional y tiene más relevancia de lo que merece en los debates sobre desarrollo y pobreza, sí puede y debe ser un elemento importante para mejorar la calidad de vida de los pobres, también en el mundo rural. En relación con las negociaciones comerciales multilaterales, hay que reconocer que no se puede vivir fuera de la OMC. Por ello, el marco de negociación de la OMC es de gran relevancia para los países en desarrollo, quienes parecen haber emprendido de forma creciente la siempre eficaz estrategia de negociar con una voz común; pero los países en desarrollo y menos desarrollados se enfrentan con reticencias a esa negociación porque han sido "consistente mente consistentes en sus demandas, mientras que los países ricos han sido consistentemente inconsistentes en sus respuestas".

El Seminario 2trató sobre "Desarrollo rural: el impacto de las políticas de ajuste y reforma en África, Asia y América”. Intervinieron Joao Guimaraes (Instituto de Estudios Sociales de La Haya), Carlos Oya (Escuela de Estudios Orientales y Africanos, Universidad de Londres) y Antonio Santamaría (Gru po de Estudios Africanos, Universidad Autónoma, Madrid). Moderó Marta Arias (Intermón - Oxfam). Simplificando un poco, podemos resumir así los mensajes allí transmitidos:

1. Asia: el caso de Vietnam, un caso de éxito. Las claves parecen haber sido la distribución de la tierra, junto con incentivos adecuados, una liberalización progresiva y una apuesta por el empleo rural no agrícola. La estrategia "exógena" de reducción de la pobreza no se ha impues to a los planes de desarrollo del país que han acabado "ganando el pulso". 
2. América: el caso de Nicaragua, un caso de fracaso. El punto de partida es distinto: existe inequidad en el acceso a la tierra y la política econé mica no se orienta a favor de los pobres. Ciertamente, se han producido avances en caracterización de la pobreza y en desarrollo institucional; la Estrategia exógena de reducción de la pobreza le ha ganado el pulso al plan nacional de desarrollo.

3. África: un caso de fracaso. Para empezar, el ajuste estructural no ha resuelto el problema. Los problemas estructurales requieren inversiones sustanciales y estrategias consistentes a largo plazo. El contexto actual (globalización y urbanización) hace inviable una agricultura basada en pequeños campesinos atomizados y sin recursos. Las estrategias de desarrollo rural deben empezar por una adecuada desagregación de la categoría de 'pobres' y medidas específicas para diferentes clases de los mismos

El Seminario 3 estuvo dedicado al tema:"Modernización de la agricultura y desarrollo rural. Los debates del mundo agrarioLas exposiciones estuvie ron a cargo de Margarita Flores (FAO México), José $\mathrm{M}^{\mathrm{a}}$ Sumpsi (Universidad Politécnica de Madrid). Moderó la sesión Eduardo Moyano, del Instituto de Estudios Sociales Avanzados de Andalucía (Córdoba). No sin cierta polémica, estas fueron las líneas de fuerza de las intervenciones:

1. Una agricultura robusta ha contribuido a un desarrollo económico sostenido en muchos países, pero va perdiendo fuerza.

2. Los agricultores de subsistencia son los guardianes de la biodiversidad y un factor clave de seguridad alimentaria: deben ser protegidos.

3. Hay que poner un énfasis continuo en seguridad alimentaria y reducción de la pobreza.

4. La sociedad moderna demanda nuevas funciones a las actividades agraria y forestal.

5. La experiencia europea señala que las economías de diversificación son más importantes que las economías de escala.

6. Se necesitan políticas de modernización agraria integradas en políticas más amplias de políticas de desarrollo rural.

7. Resulta demagógico hablar de que se está tendiendo hacia el De sarrollo Rural cuando se dedica menos dinero en los presupuestos comunitarios. 
Al final quedó en el aire una doble perspectiva, aparentemente contradi€ toria: "para los países de bajos ingresos con alta proporción de población rural, si la agricultura no es el motor de desarrollo, ¿cuál es ese motor?" (M. Flores); "las zonas rurales con mayor crecimiento son las que tienen su eco nomía más diversificada... ¿Sólo agricultura?, ni de broma” (J.Ma Sumpsi).

"Desarrollo rural y territorio. Elementos y actores protagonistas del desa rrollo rural" fue el tema desarrollado en elSeminario 4. Intervinieron Mayra Falck (Universidad Zamorano, Honduras) y Germán Escobar (RIMISP, Chile). Moderó José J. Romero (Fundación ETEA, Córdoba). De nuevo, aun a riesgo de simplificar, destacamos las siguientes ideas surgidas de las exposiciones y del debate subsiguiente.

1. El enfoque territorial del desarrollo rural gana peso progresivamente aunque las visiones y actuaciones son muy diversas.

2. La posibilidad de integrar esfuerzos de los creadores de riqueza (sector privado) con los facilitadores de procesos y proveedores de bienes públicos (organismos y servicios del Estado) es un avance significativo frente a otros esquemas y modelos de políticas diseñados de arriba hacia abajo y puestos en marcha a través de programas sectoriales centralizados.

3. Son factores críticos:

- La selección de territorios estratégicos.

- La fortaleza de la institucionalidad en el territorio.

- Operar en los territorios a diferentes niveles y con todas las unidades sociales con el fin de garantizar que se atiende la problemática en su conjunto.

- Trabajar en alianza con actores con potencial, capaces de aprender y generar difusión del conocimiento.

- Crear una visión compartida del territorio que genere cohesión.

- No olvidar que es importante promover impacto de corto plazo y efecto demostrativo.

- Fortalecer los activos o capitales mediante el desarrollo de nuevas formas de oferta técnica apropiadas a los territorios, con adecuadas estrategias de comunicación y educación. 
- La dificultad de gobiernos y de la cooperación internacional para dar el salto desde las visiones sectoriales al enfoque territorial.

El Seminario 5 estuvo centrado en el "Análisis de casos de Desarrollo rural: Vietnam, Nicaragua, Suráfrica". Los expositores fueron Pham Quang Dieu (NIAPP, Vietnam), Fátima Ismael (CAFENICA, Nicaragua) y Colette So lomon (Women on Farm, Suráfrica). Moderó Ignacio Trueba (Universidad Politécnica de Madrid).

1. En Vietnam los cambios estructurales han provocado cambios es pectaculares en las variables macroeconómicas y en la producción agraria.

2. En Nicaragua, las experiencias de SOPPEXCCA y de CAFENICA demues tran la viabilidad de organizaciones y actividades productivas bajo un enfoque de empresas sociales de los pequeños productores.

3. En Sudáfrica, la ONG "Women on farms" está consiguiendo importantes logros en la lucha por el empoderamiento de las mujeres rurales y el cambio de las estructuras legales y laborales discriminatorias.

Si hubiera que resaltar, en una síntesis atrevida de todo el debate susci tado en esta línea temática 5 dedicada al desarrollo rural, subrayaríamos algunas conclusiones bajo la forma de los siguientes consensos y debates abiertos.

1. Consenso acerca de la importancia de la lucha contra la pobreza en el medio rural. La urgencia del problema obliga a revisar paradigmas y a evitar generalizaciones.

2. Debate acerca del papel que las actividades agrarias y no agrarias deben jugar en el desarrollo del medio rural. Debate sobre la preponderancia o no del empleo rural agrario sobre el empleo rural no agrario.

3. Debate acerca de la importancia que el comercio tiene en el desarrollo de los países y en el mundo rural. El comercio internacional no es la única clave de desarrollo pero es un factor relevante. No parece haber más alternativas a la OMC que una OMC en la que los temas y las ne gociaciones se conduzcan de manera distinta.

4. Consenso sobre la necesidad de que el ajuste estructural y las estra tegias de reducción de la pobreza (que es más ajuste) se apoyen en condiciones previas que fomenten la equidad en el acceso a los recursos en el mundo rural para asegurar su eficacia. 
5. Consenso acerca de cómo las políticas de ajuste estructural han sido escasamente estructurales. Su impacto varía en función de la capacidad endógena para generar políticas pro-pobres y del grado de autonomía en la definición de sus prioridades y políticas económicas.

6. Consenso acerca de la pertinencia y viabilidad del modelo de desarrollo territorial rural. Debate acerca de las condiciones que hacen posible su éxito, en particular en relación a la institucionalidad y el apoyo público.

Para terminar, destacamos que al organizar un Congreso de este alto nivel, el Ayuntamiento de Madrid ha dado muestras de visión política amplia; se comentaba en los pasillos que el tono general de las intervenciones no iba precisamente en la línea política del partido que gobierna en esa gran institu ción. A los responsables de su programa de Cooperación hay que reconocerles su ambición y su amplitud de miras en el planteamiento del mismo. 